\title{
Polyhedral properties for the intersection of two knapsacks
}

\author{
Quentin Louveaux* $\quad$ Robert Weismantel*
}

August 2, 2006

\begin{abstract}
We address the question to what extent polyhedral knowledge about individual knapsack constraints suffices or lacks to describe the convex hull of the binary solutions to their intersection. It turns out that the sign patterns of the weight vectors are responsible for the types of combinatorial valid inequalities appearing in the description of the convex hull of the intersection. In particular, we introduce the notion of an incomplete set inequality which is based on a combinatorial principle for the intersection of two knapsacks. We outline schemes to compute nontrivial bounds for the strength of such inequalities w.r.t. the intersection of the convex hulls of the initial knapsacks. An extension of the inequalities to the mixed case is also given. This opens up the possibility to use the inequalities in an arbitrary simplex tableau.
\end{abstract}

\section{Introduction}

Starting with the basic research on knapsack polyhedra in the seventies by [1], [4] and [9], many papers have emerged in the past that deal with the polyhedral structure of knapsack problems. The interest in the combinatorics of a binary knapsack problem is justified by the fact that every general integer programming problem can be described as the intersection of a finite number of knapsack problems. As a starting point, it is clear that principle investigations about knapsack problems automatically provide insight into more general integer programming polyhedra. A study of the substantial literature on binary knapsack polyhedra also reveals that linear inequalities for such a special integer program are based on covers, i.e., subsets of variables such that the sum of the associated weights in the knapsack constraint exceeds the given capacity. In other words, a cover is a forbidden substructure or a minor whose presence must be prohibited by linear constraints. One basic observation is that the sum

*Address: Otto-von-Guericke-Universität Magdeburg, Department of Mathematics/IMO, Universitätsplatz 2, 39106 Magdeburg, Germany. Email: \{louveaux, weismant\}@mail. math.uni-magdeburg.de 
of the variables in such a cover can be at most the cardinality of the cover minus 1. More general techniques such as extended weight inequalities may be used to express further linear constraints associated with covers, see [8].

Given that fact that a description for knapsack problems is based on such a simple principle like covers, it is quite natural to ask whether or not there are other geometric or combinatorial principles that play a role for binary integer programs when several constraints are to be considered simultaneously. Little research in this direction has been carried out. In [7] extended weight inequalities for the single knapsack problem have been generalized to multiple constraints with nonnegative coefficients. Fernandez and Jørnsten [2] show the existence of cover type inequalities when a $\leq$-constraint is intersected with a $\geq$ constraint. Günlük and Pochet [3] propose the principle of mixing for a specific mixed-integer model.

The fundamental geometric difference between the single knapsack constraint and the presence of several constraints is that in the latter case incomparabilities between the weight vectors occur. These incomparabilities between the weight vectors are reflected in a new substructure that we refer to as an "incomplete set" that is responsible for new types of constraints that cannot be explained with cover- and knapsack type constraints.

The following notation is used throughout the paper. Given integer data $a_{i}, b_{i} \in \mathbb{Z}$ for $i=0, \ldots, n$ we define the set of feasible solutions to the first and second knapsack constraint by $X_{1}$ and $X_{2}$, respectively. $X$ denotes the integer points lying in the intersection of the two constraints.

$$
\begin{aligned}
& X_{1}=\left\{x \in\{0,1\}^{n}: \sum_{i=1}^{n} a_{i} x_{i} \leq a_{0}\right\}, \\
& X_{2}=\left\{x \in\{0,1\}^{n}: \sum_{i=1}^{n} b_{i} x_{i} \leq b_{0}\right\}, \\
& X=\left\{x \in\{0,1\}^{n}: x \in X_{1} \cap X_{2}\right\} .
\end{aligned}
$$

It is well known that in general we have that $\operatorname{conv}(X) \neq \operatorname{conv}\left(X_{1}\right) \cap \operatorname{conv}\left(X_{2}\right)$. Without loss of generality we can assume that $a_{i} \geq 0$. If this condition does not hold, then we can complement variables so as to meet this requirement. Therefore, the set of variables $N=\{1, \ldots, n\}$ can always be partitioned into sets $N_{+}$and $N_{-}$such that

$$
N_{+}=\left\{i \in N \mid a_{i} \geq 0, b_{i} \geq 0\right\} \text { and } N_{-}=\left\{i \in N \mid a_{i} \geq 0, b_{i}<0\right\} .
$$

It turns out that the sign pattern for the two dimensional vectors $\left(a_{i}, b_{i}\right)$ is essentially responsible for the types of combinatorial valid inequalities appearing in the description of $\operatorname{conv}(X)$.

The paper is organized as follows. We begin our discussions in Section 2 with an extension of cover constraints when the feasible region is described by the intersection of two constraints in binary variables. The main difficulty is that in the presence of two constraints with different sign patterns for the 
column vectors, the corresponding set of binary solutions satisfying the two constraints simultaneously does not define an independence system. Hence, covers or circuits of the system may not always exist. We prove in particular that when the second constraint has only weights plus-minus-one, then all forbidden minors for the intersection arise from covers for a conic combination of the two constraints. However, in the general case of arbitrary weight values, there exist forbidden minors for the intersection which do not correspond to covers for any conic combination of the two constraints. This illustrates an essential difference between polyhedra associated with integer programs defined by the intersection of several constraints and the single knapsack problem. Section 3 deals with a general combinatorial inequality that plays an important role for the intersection of several constraints. It is based on the concept of so-called incomplete sets, i.e., sets of columns that cannot be simultaneously set to one unless a certain subset of other items is also set to one. It turns out that these inequalities are under mild assumptions very strong. The strength of such inequalities can be computed with several constructions that we outline in Section 4. Section 5 discusses extensions to the mixed integer scenario.

\section{Forbidden minors for the intersection of two knapsacks}

In this section we investigate the role that the sign patterns of the column vectors in two knapsack constraints play in the derivation of combinatorial valid inequalities.

For the single knapsack problem, an important class of inequalities consists of the cover inequalities. A cover for a knapsack constraint is a subset of items whose total weight exceeds the capacity. Hence, a cover is a forbidden minor for a single knapsack constraint whose existence must be forbidden in terms of a linear inequality. If $C$ denotes the cover, then such a cover constraint requires that

$$
\sum_{j \in C} x_{j} \leq|C|-1 .
$$

If $C$ is minimal, then $C$ defines a circuit of the independence system defined by all the feasible solutions to the single knapsack problem. In the following we discuss the existence of such "forbidden minor"inequalities for the case of the intersection of two knapsacks. The main difficulty is that in the presence of two constraints with different sign patterns for the column vectors, the corresponding set of binary solutions satisfying the two constraints simultaneously does not define an independence system. Hence, circuits do not exist.

In particular if such inequalities exist, we would like to know whether they can be found in one of the two knapsacks or as valid inequalities for a conic combination of the two knapsacks. We now consider valid inequalities of the type

$$
\sum_{i \in C} x_{i} \leq|C|-1 .
$$


We start with a simple observation. Consider the problem of finding $x \in X=$ $X_{1} \cap X_{2}$ with $X_{1}=\left\{x \in\{0,1\}^{n}: \sum_{i=1}^{n} a_{i} x_{i} \leq a_{0}\right\}$ and $X_{2}=\left\{x \in\{0,1\}^{n}\right.$ : $\left.\sum_{i=1}^{n} b_{i} x_{i} \leq b_{0}\right\}$.

Observation 1 Suppose that $N_{-}=\emptyset$, i.e., $a_{i} \geq 0$ and $b_{i} \geq 0$ for $i=1, \ldots, n$. Let the inequality

$$
\sum_{i \in C} x_{i} \leq|C|-1
$$

be valid for all points in $X$. Then (5) is either valid for $X_{1}$ or for $X_{2}$.

Proof: If (5) is valid for $X$, then $C$ is not a feasible set for $X$. Since $N_{-}=\emptyset$, it follows that every true superset of $C$ is also not feasible for $X$. Moreover, since $C$ is not feasible for $X, C$ is not feasible for $X_{1}$ or it is not feasible for $X_{2}$. By definition, $C$ defines a cover for the corresponding knapsack constraint.

This observation is not true when some coefficients are negative as the following example shows. This is a first indication that the derivation of valid inequalities for the intersection of two constraints gets significantly more complicated in the presence of both positive and negative coeffficients in the second knapsack constraint. In fact, sign patterns of the weight vectors influence the inequality description quite substantially. More precisely, the next examples illustrate that there exist valid inequalities for $X$ of the form

$$
\sum_{i \in C} x_{i} \leq|C|-1
$$

with $C \subseteq N$ such that $C$ is neither a cover for $X_{1}$ nor a cover for $X_{2}$. Only the simultaneous consideration of the two constraints allows us to derive this inequality.

Example 1 Consider the problem

$$
\begin{aligned}
X=\left\{x \in\{0,1\}^{2}: x_{1}+x_{2}\right. & \leq 1 \\
x_{1}-x_{2} & \leq 0 \quad\} .
\end{aligned}
$$

We have that $X=\{(0,0),(0,1)\}$. Therefore $x_{1} \leq 0$ is valid for $X$. On the other hand the inequality is not valid for a binary set defined by (6) or (7) only.

We can also present a slightly more elaborate example.

Example 2 Consider the problem

$$
\left.\begin{array}{r}
X=\left\{x \in\{0,1\}^{8}: 13 x_{1}+11 x_{2}+24 x_{3}+19 x_{4}+29 x_{5}+33 x_{6}+21 x_{7}+18 x_{8} \leq 78\right. \\
x_{1}+x_{2}+x_{3}+x_{4}-x_{5}-x_{6}-x_{7}-x_{8} \leq 0
\end{array}\right\} .
$$

In this example we have that $N_{+}=\{1,2,3,4\}$ and $N_{-}=\{5,6,7,8\}$. The inequality

$$
x_{3}+x_{4} \leq 1
$$


is valid for $X$. Indeed if we want a feasible solution with $x_{3}=x_{4}=1$, then we need at least two variables from $\{5,6,7,8\}$ set to one in order to satisfy (9). But then the value obtained in (8) is at least 82 which exceeds the capacity. Therefore no solution includes $x_{3}=x_{4}=1$. On the other hand, it is clear that (10) is neither valid for $X_{1}$ nor for $X_{2}$ since it is easy to construct feasible solutions including $x_{3}=x_{4}=1$ for both single knapsacks.

These examples illustrate that the presence of negative coefficients in the second constraint leads to simple inequalities that cannot be derived by the analysis of a single constraint. It is however possible in these examples to deduce valid inequalities by considering a conic combination of the two initial inequalities. The following quite general statement can be made. The proof follows a suggestion made by one anonymous referee.

Theorem 1 Let $X=X_{1} \cap X_{2}$, with

$$
\begin{aligned}
& X_{1}=\left\{x \in\{0,1\}^{n}: \sum_{i=1}^{n} a_{i} x_{i} \leq a_{0}\right\}, \\
& X_{2}=\left\{x \in\{0,1\}^{n}: \sum_{i=1}^{n} b_{i} x_{i} \leq b_{0}\right\},
\end{aligned}
$$

with $a_{i} \in \mathbb{Z}_{+}, b_{i} \in \mathbb{Z}$. Let $J \subseteq\{1, \ldots, n\}$ be such that the inequality

$$
\sum_{j \in J} x_{j} \leq|J|-1
$$

is valid for $X$. If $b_{i} \in\{-1,0,1\}$ for all $i=1, \ldots, n$, then there exist conic multipliers $u, v \geq 0$ such that (13) is valid for the single knapsack set $X(u, v)=$ $\left\{x \in\{0,1\}^{n}: x\right.$ satisfies $\left.u(11)+v(12)\right\}$.

Proof: We consider the linear relaxations of $X_{1}$ and $X_{2}$ that we denote by $P_{1}$ and $P_{2}$ respectively. Note that following our assumptions $b_{i} \in\{-1,0,1\}$, we have that $b=\left(b_{1}, \ldots, b_{n}\right)$ is totally unimodular and therefore $P_{2}=\operatorname{conv}\left(X_{2}\right)$. In particular, every vertex of $P_{2}$ is integer. Consider then $F=\left\{x \in\{0,1\}^{n}\right.$ : $\left.\sum_{i \in J} x_{i}=|J|\right\}$. We claim that $F \cap\left(P_{1} \cap P_{2}\right)=\emptyset$. Let $p \in F \cap\left(P_{1} \cap P_{2}\right) \neq \emptyset$, by contradiction. Since $p \in P_{2}$, it can be written as a nontrivial convex combination of vertices $v^{1}, \ldots, v^{k}$ of $P_{2}$. Notice that since $\sum_{i \in J} x_{i} \leq|J|$ is valid for $P_{2}$, all vertices $v^{1}, \ldots, v^{k}$ must be in $F$. In fact, we now have that $v^{i} \in F \cap P_{2}$ for all $i$. Moreover $p \in F \cap P_{1} \cap P_{2}$, i.e., $p$ satisfies $a^{T} p \leq a_{0}$. Since $p$ is a convex combination of the $v^{i}$, it follows that at least one of the $v^{i}, v^{i_{0}}$ say, satisfies $a^{T} v^{i_{0}} \leq a_{0}$. This implies that $v^{i_{0}} \in F \cap P_{1} \cap P_{2}$. But since the vertices of $P_{2}$ are integer, this is a contradiction with the validity of (13). Hence $F \cap\left(P_{1} \cap P_{2}\right)=\emptyset$. 
It implies that the system

$$
\begin{aligned}
a^{T} x & \leq a_{0} \\
b^{T} x & \leq b_{0} \\
-\sum_{i \in J} x_{i} & \leq-|J| \\
x_{i} & \leq 1 \quad i=1, \ldots, n
\end{aligned}
$$

is infeasible. Hence by the Farkas Lemma we can find multipliers $u, v, w, s_{i} \geq$ $0, i=1, \ldots, n$ corresponding to $(14),(15),(16),(17)$ respectively such that

$$
\begin{aligned}
& (u a+v b)+\sum_{i=1}^{n} s_{i} e_{i} \geq w \operatorname{supp}(J), \\
& \left(u a_{0}+v b_{0}\right)+\sum_{i=1}^{n} s_{i}<w|J|
\end{aligned}
$$

where $\operatorname{supp}(J)$ is the support vector of $J$ and $e_{i}$ is the $i-t h$ unit vector. We claim that $u, v$ are the desired multipliers. Consider $x \in X(u, v)$ and multiply (18) by $x$. We obtain

$$
\begin{aligned}
w \sum_{i \in J} x_{i} & \leq(u a+v b)^{T} x+\sum_{i=1}^{n} s_{i} x_{i} & & \text { by }(18) \\
& \leq\left(u a_{0}+v b_{0}\right)+\sum_{i=1}^{n} s_{i} & & \text { since } x \in X(u, v) \\
& <w|J| & & \text { by }(19) .
\end{aligned}
$$

Therefore, we have for all $x \in X(u, v)$, since $w \geq 0$ (and obviously $w \neq 0$ ),

$$
\sum_{i \in J} x_{i}<|J|
$$

which implies the result for every integer point $x \in X(u, v)$.

Example 2 continued Consider again the set $X$ defined by the constraints (8) and (9) in Example 2. We compute the combination (8)+18(9) of the two constraints. This leads to the inequality

$$
31 x_{1}+29 x_{2}+42 x_{3}+37 x_{4}+11 x_{5}+15 x_{6}+3 x_{7}+0 x_{8} \leq 78
$$

which is therefore satisfied by all points in $X$. For this knapsack, we remark that $\{3,4\}$ is a cover. In other words, $x_{3}+x_{4} \leq 1$ is a valid inequality for a conic combination of the two initial constraints.

Since in our initial example the constraint $x_{3}+x_{4} \leq 1$ defines a cover for an aggregated knapsack constraint, one might be tempted to think that Theorem 
1 can be further extended so as to consider general weight vectors in the second constraint instead of the special values \pm 1 . This extension is however not true. It can happen that a simple combinatorial cover type inequality is valid for the intersection of two constraints while the same inequality is not valid for any conic combination of the two constraints. We prove this result next.

Theorem 2 There exist instances defining $X, X_{1}, X_{2}$ according to (1), (2) and (3) for which there exist valid inequalities for $X$ of the form

$$
\sum_{j \in J} x_{j} \leq|J|-1
$$

which do not define a cover constraint for all valid single knapsack relaxations of $X$. In particular, for every $u, v \geq 0$, the inequality $\sum_{j \in J} x_{j} \leq|J|-1$ is not valid for

$$
X(u, v)=\left\{x \in\{0,1\}^{n}: \sum_{i=1}^{n}\left(u a_{i}+v b_{i}\right) x_{i} \leq u a_{0}+v b_{0}\right\} .
$$

Proof: Consider the set

$$
\begin{aligned}
X=\left\{x \in\{0,1\}^{2}:\right. & 2 x_{1}+x_{2} \leq 2 \\
- & \left.2 x_{1}+x_{2} \leq 0\right\} .
\end{aligned}
$$

By simple enumeration we obtain $X=\{(0,0),(1,0)\}$. In particular the inequality $x_{2} \leq 0$ is valid. We next show that it is not valid for any knapsack obtained by taking a conic combination of the two constraints (21) and (22). Let $u, v \geq 0$ be real multipliers and

$$
X(u, v)=\left\{x \in\{0,1\}^{2}:(2 u-2 v) x_{1}+(u+v) x_{2} \leq 2 u\right\} .
$$

We now show that for all $u, v \geq 0$, there exists at least one solution of $X(u, v)$ with $x_{2}=1$.

If $v \leq u$ we clearly have $(0,1) \in X(u, v)$.

If $v>u,(1,1) \in X(u, v)$. Indeed by adding the two coefficients, we obtain $3 u-v$ which, by hypothesis, satisfies $3 u-v<2 u$.

Hence we conclude that $x_{2} \leq 0$ is never valid for $X(u, v)$.

Theorem 2 makes precise that for the intersection of even two constraints with arbitrary sign patterns, forbidden minors exist that never define circuits of the independence system defined by the set of binary solutions to a single knapsack relaxation. This implies that cutting planes based on single knapsack polyhedra are not sufficient to tackle binary programs with many constraints. The polyhedral situation in the presence of several constraints is however much more complex. In the next section we present a general principle that allows us to derive valid inequalities for programs with several constraints. The principle is simple to state, but algorithmically quite difficult to detect. It is based on an incomplete set of items, i.e., a subset of variables that cannot be simultaneously set to one unless a second distinct set of items is also set to one. 


\section{$3 \quad$ Incomplete set inequalities}

Recall that we consider the binary program

$$
\begin{aligned}
& X_{1}=\left\{x \in\{0,1\}^{n}: \sum_{i=1}^{n} a_{i} x_{i} \leq a_{0}\right\}, \\
& X_{2}=\left\{x \in\{0,1\}^{n}: \sum_{i=1}^{n} b_{i} x_{i} \leq b_{0}\right\}, \\
& X=\left\{x \in\{0,1\}^{n}: x \in X_{1} \cap X_{2}\right\},
\end{aligned}
$$

with the set of variables $N=\{1, \ldots, n\}$ being partitioned into sets

$$
N_{+}=\left\{i \in N \mid a_{i} \geq 0, b_{i} \geq 0\right\} \text { and } N_{-}=\left\{i \in N \mid a_{i} \geq 0, b_{i}<0\right\} .
$$

In the following, we use the convenient notation $a(T)=\sum_{i \in T} a_{i}$, and $b(T)=$ $\sum_{i \in T} b_{i}$ for some subset $T \subseteq N_{+} \cup N_{-}$. We now introduce the basic notion to derive valid inequalities for $X$.

Definition 1 Let $I \subseteq N_{+} \cup N_{-}$. We call $I$ an incomplete set if $a(I) \leq a_{0}$ and $b(I)>b_{0}$. Along with an incomplete set $I$, we introduce the quantities

$$
\begin{aligned}
& r(I)=a_{0}-a(I) \\
& e(I)=b(I)-b_{0},
\end{aligned}
$$

called the residue and the excess of the incomplete set, respectively.

The name "incomplete set" comes from the fact that $I$ is infeasible on its own but could be made feasible by setting appropriate variables of $N_{-} \backslash I$ to 1 .

Example 3 Consider the problem

$$
\left.\begin{array}{rl}
X=\left\{x \in\{0,1\}^{7}: x_{1}+3 x_{2}+2 x_{3}+2 x_{4}+3 x_{5}+2 x_{6}+2 x_{7}\right. & \leq 12 \\
3 x_{1}+2 x_{2}-x_{3}-x_{5}-3 x_{6}-2 x_{7} & \leq 1
\end{array}\right\}
$$

Let us consider $I=\{1,2,3,4\}$. We see that $r(I)=4$ and $e(I)=3$. The set $I$ is represented in Figure 1, each variable being represented by its corresponding vector. Note that because we have a canonical form, all the vectors point to the right and either upward (for the columns in $N_{+}$) or downward (for the columns in $N_{-}$). In the figure, the residue and the excess of the chosen set are illustrated. This means that, to turn $I$ into a feasible set, we have to find (an)other vector(s) whose sum lies in the box indicated as the "feasible box". In the example shown in Figure 2, we must select at least item 6 to complete $I$ to a feasible set. It follows that a valid inequality for $X$ is

$$
x_{1}+x_{2}+x_{3}+x_{4}-x_{6} \leq 3 .
$$




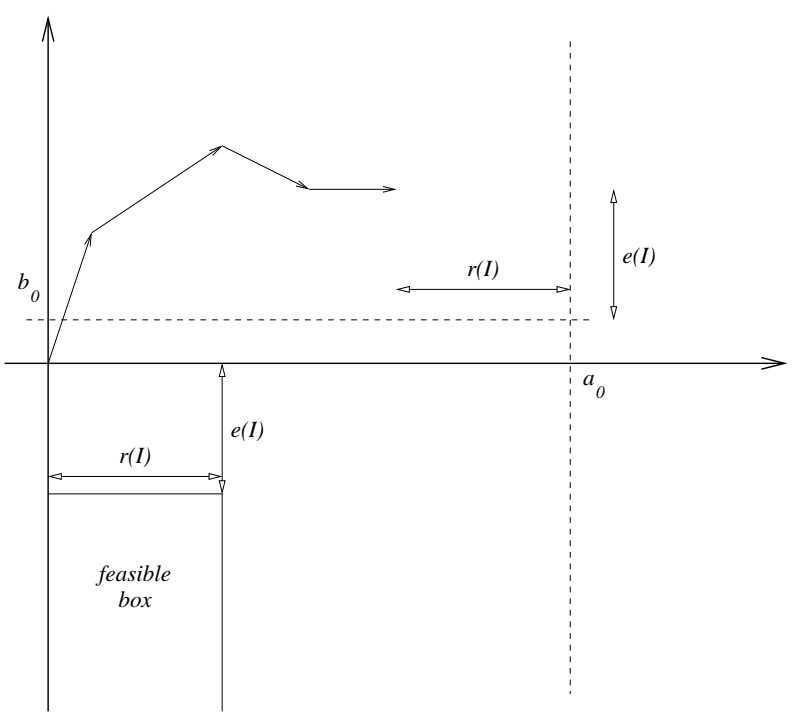

Figure 1: The geometry of incomplete sets

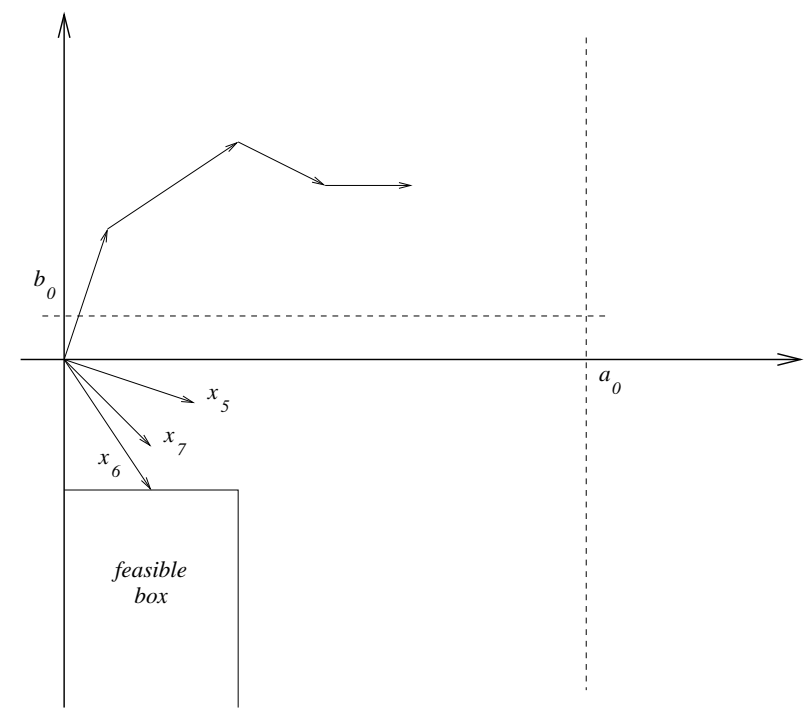

Figure 2: We must take at least $x_{6}$ to complete $I$ into a feasible set 
Let us now explain more generally how we derive an incomplete set inequality. We start with an incomplete set $I$ and its associated residue $r(I)$ and excess $e(I)$. We then consider the subproblem

$$
\begin{aligned}
P_{I}=\left\{x \in\{0,1\}^{t}:\right. & \sum_{j \in N_{-} \backslash I} a_{j} x_{j} \leq r(I) \\
& \left.\sum_{j \in N_{-} \backslash I}-b_{j} x_{j} \geq e(I) \quad\right\},
\end{aligned}
$$

where $t=\left|N_{-} \backslash I\right|$. The next proposition indicates how a valid inequality can be generated from $I$ and the set of solutions of $P_{I}$. We suppose that the solutions in $P_{I}$ are represented as the columns of a matrix. We first need to make precise the notion of covering.

Definition 2 Let $A \in\{0,1\}^{n \times m}$ be a binary matrix. We call $C \subseteq\{1, \ldots, m\}$ $a$ covering of $A$ if

$$
\sum_{i \in C} A_{i} . \geq \mathbf{1}
$$

with 1 being an $m$-dimensional row vector.

For the ease of notation, we define, if $P_{I}=\emptyset$, that $\emptyset$ is a covering of $P_{I}$.

Proposition 1 Let $I$ be an incomplete set for $X$, and $P_{I}$ be the set of solutions of the corresponding subproblem. Let $I^{C}$ be any covering of $P_{I}$, i.e., $\sum_{i \in I^{C}} x_{i} \geq$ 1 is valid for $P_{I}$. Then the inequality

$$
\sum_{i \in I} x_{i}-\sum_{j \in I^{C}} x_{j} \leq|I|-1
$$

is valid for $X$.

Proof: The only way that inequality (23) can be violated is if there exists some valid point $x \in X$ with $x_{i}=1$ for all $i \in I$ and $x_{j}=0$ for all $j \in I^{C}$. But this is impossible by the construction of $P_{I}$ and $I^{C}$.

In the special case where $N_{+}$is empty, a particular subfamily of incomplete set inequalities $\left(I^{C}=\emptyset\right)$ have been introduced in [2]. We remark that the authors use the name partial cover instead of incomplete set.

Proposition 1 has a very simple interpretation. Suppose that we fix the value of the variables in $I$ to 1 . By computing the set $P_{I}$, we try to search for all the possibilities to complete $I$ into a feasible set. An inequality of the type (23) indicates that if all the variables of $I$ are set to 1 , then at least one of the variables from $I^{C}$ has to be set to 1 .

Example 2 (continued) Consider the set

$$
\begin{array}{r}
X=\left\{x \in\{0,1\}^{8}: 13 x_{1}+11 x_{2}+24 x_{3}+19 x_{4}+29 x_{5}+33 x_{6}+21 x_{7}+18 x_{8} \leq 78\right. \\
\left.x_{1}+x_{2}+x_{3}+x_{4}-x_{5}-x_{6}-x_{7}-x_{8} \leq 0\right\} .
\end{array}
$$


Let us first consider the incomplete set $I_{1}=\{3,4\}$. We compute

$$
r\left(I_{1}\right)=35, e\left(I_{1}\right)=2 .
$$

It is readily verified that $P_{I_{1}}=\emptyset$. Therefore

$$
x_{3}+x_{4} \leq 1
$$

is satisfied by all the points in $X_{2}$. It can be verified that it actually defines a facet of $\operatorname{conv}(X)$. We then consider $I_{2}=\{2,4\}$. We compute the residue and the excess and obtain

$$
r\left(I_{2}\right)=48, e\left(I_{2}\right)=2 .
$$

It can be verified that $P_{I_{2}}=\left\{e_{5}+e_{8}, e_{7}+e_{8}\right\}$. Two ways to minimally cover $P_{I_{2}}$ are either $I_{1}^{C}=\{8\}$ or $I_{2}^{C}=\{5,7\}$. Therefore the inequalities

$$
\begin{array}{r}
x_{2}+x_{4}-x_{8} \leq 1 \\
x_{2}+x_{4}-x_{5}-x_{7} \leq 1
\end{array}
$$

are valid incomplete set inequalities for $X$.

\section{The strength of incomplete set inequalities}

In the previous sections we made an attempt to shed some light on the question how far one can go using polyhedral information about single knapsack polyhedra for a binary optimization problem with two constraints. The family of incomplete set inequalities are indeed based on a principle that applies to several original constraints in contrast to cover inequalities based on a one dimensional constraint.

This suggests to tackle binary problems with several constraints incorporating incomplete set inequalities. The problem, however, is that it seems quite involved to come up with efficient general heuristic schemes for the cut generation. We believe that such a cut generation tool must be specialized towards specific families of instances. We refrain from going further into this topic here. Instead we want to give further evidence how strong these inequalities can be compared with the single knapsack relaxations. To demonstrate this effect we have randomly generated a series of binary instances with twelve variables and two nontrivial constraints whose coefficients are in the interval $[-15,15]$. For each such instance we first computed separately the inequality description for the convex hull of all binary solutions associated with each of the two initial constraints. We then selected an incomplete set inequality and generated a strongly perturbed objective function from the corresponding normal vector. Next we compare the bound provided by the lower and upper bounds of the variables together with the two initial constraints and the incomplete set inequality with the bound given by the intersection of the two convex hulls and the true integer optimum. The results are surprising - at least for us. Table 1 presents a selection of our tests on one instance with three different objective functions. 


\begin{tabular}{ll}
\hline $\begin{array}{l}\text { incomplete set inequality } \\
\text { objective }\end{array}$ & $\begin{array}{l}x_{1}+x_{3}+x_{5}-x_{11} \leq 2 \\
\\
\end{array}$ \\
& \multicolumn{1}{c}{$+28 x_{1}+171 x_{3}+314 x_{5}-99 x_{11}-73 x_{4}-19 x_{12}+31 x_{10}$} \\
\hline IP optimum & 516 \\
LP bound w/ inc. set ineq. & 538 \\
LP bound w/ 2 convex hulls & 568 \\
Initial LP bound & 641 \\
\hline \hline incomplete set inequality & $x_{1}+x_{3}+x_{8}+x_{11} \leq 3$ \\
objective & $2000 x_{1}+2101 x_{3}+2151 x_{8}+2272 x_{11}$ \\
& \multicolumn{1}{c}{$+512 x_{2}+733 x_{5}+333 x_{9}+121 x_{10}$} \\
\hline IP optimum & 7257 \\
LP bound w/ inc. set ineq. & 7475 \\
LP bound w/ 2 convex hulls & 7968 \\
initial LP bound & 8668 \\
\hline \hline incomplete set inequality & $x_{3}+x_{5}+x_{6}-x_{12} \leq 2$ \\
objective & $11287 x_{3}+15690 x_{5}+12003 x_{6}-17123 x_{12}$ \\
& \multicolumn{1}{c}{$+4000 x_{2}+3459 x_{11}-1212 x_{9}+3001 x_{7}$} \\
\hline IP optimum & 35152 \\
LP bound w/ inc. set ineq. & 36619 \\
LP bound w/ 2 convex hulls & 36795 \\
initial LP bound & 43006 \\
\hline
\end{tabular}

Table 1: Comparison of the performance of an incomplete set inequality vs. adding all the facets of $\operatorname{conv}\left(X_{1}\right)$ and $\operatorname{conv}\left(X_{2}\right)$ to the formulation wrt. a perturbed objective 
In all these cases the bound given by one incomplete set inequality and the original LP formulation outperforms the bound provided by taking the full description of the two convex hulls in account. (The latter formulation roughly includes 4000 constraints)! We conclude from these tests that incomplete set inequalities seem to have a good effect on the quality of a formulation. In order to support this hypothesis we next develop a tool to analyze the strength of a given incomplete set inequality

$$
\sum_{i \in I} x_{i}-\sum_{j \in I^{C}} x_{j} \leq|I|-1 .
$$

In particular we investigate how strong it is to use the inequalities obtained by both knapsacks separately compared to one incomplete set inequality. Therefore we focus on the program

$$
\begin{aligned}
& \max \sum_{i \in I} x_{i}-\sum_{j \in I^{C}} x_{j} \\
& \text { s.t. } x \in \operatorname{conv}\left(X_{1}\right) \cap \operatorname{conv}\left(X_{2}\right),
\end{aligned}
$$

where $X_{1}=\left\{x \in\{0,1\}^{n}: \sum_{i \in N} a_{i} x_{i} \leq a_{0}\right\}$ corresponds to the first inequality and $X_{2}=\left\{x \in\{0,1\}^{n}: \sum_{i \in N_{+}} b_{i} x_{i}-\sum_{j \in N_{-}} b_{j} x_{j} \leq b_{0}\right\}$ corresponds to the second inequality.

By definition the value of the linear program (29) is bounded above by $|I|$. We assume that the inequality is tight such that the value of the linear program is bounded below by $|I|-1$. Therefore it is natural to define the strength of an incomplete set inequality, with respect to the two knapsacks taken separately as

$$
s\left(I, I^{C}\right)=\max \left\{\sum_{i \in I} x_{i}-\sum_{j \in I^{C}} x_{j}: x \in \operatorname{conv}\left(X_{1}\right) \cap \operatorname{conv}\left(X_{2}\right)\right\}-(|I|-1) .
$$

A value of $s\left(I, I^{C}\right)$ close to 1 indicates that the inequality is strong and that it reflects information that is not contained in the knapsacks taken separately. Table 2 provides us with the information about the strength of all the incomplete set inequalities that define facets for the instance analyzed in Table 1. In total we

\begin{tabular}{c|cccc} 
strength & 0 & 0.5 & 0.667 & 0.75 \\
\hline \# facets & 12 & 20 & 7 & 2 \\
\% facets & $29 \%$ & $49 \%$ & $17 \%$ & $5 \%$ \\
\hline
\end{tabular}

Table 2: Percentage of the incomplete set type facets that reach a given strength

have 41 inequalities with \pm 1 -coefficients that meet our definition of an incomplete set inequality or correspond to a cover inequality. $29 \%$ of these inequalities are indeed already valid for one of the two knapsack relaxations. By definition, their strength is zero. The majority of these inequalities have a strength of 0.5 . 
The remaining ones have a strength of even more than two thirds. The fact that the strength of these inequalities seems to be surprisingly high in this example, motivates us to analyze this question further. Unfortunately, the optimization problem (29) is extremely difficult to solve as it requires two convex hull representations. This leads us to develop analytically strong bounds on the strength of inequality (28). Throughout we use the notation $A \in X_{1}$, where $A$ is a set of indices, to describe the fact that $x \in X_{1}$ with $x_{i}=1$ for all $i \in A, x_{j}=0$ for all $j \notin A$. We start with an example.

Example 4 Consider

$$
\begin{array}{r}
X=\left\{x \in\{0,1\}^{9}: 3 x_{1}+6 x_{2}+6 x_{3}+2 x_{4}+7 x_{5}+3 x_{6}+8 x_{7}+6 x_{8}+10 x_{9} \leq 34\right. \\
\left.7 x_{1}+7 x_{2}+9 x_{3}-10 x_{5}-4 x_{6}-x_{7}-3 x_{8}-3 x_{9} \leq 0\right\} .
\end{array}
$$

It can be checked that

$$
x_{1}+x_{3}+x_{4}+x_{7} \leq 3
$$

is valid for $X$. We denote $I=\{1,3,4,7\}$. We now show that $s(I) \geq 1 / 2$. To do this we find a point $x \in \operatorname{conv}\left(X_{1}\right) \cap \operatorname{conv}\left(X_{2}\right)$ with $x_{1}+x_{3}+x_{4}+x_{7}=3.5$.

Let us remark that (32) is not valid for (31) and $F=\{5,6,8\}$ is a set such that $I \cup F \in X_{2}$. Of course we have that $I \cup F \notin X_{1}$. On the other hand, it suffices to remove an element from $I$ in order to obtain a solution for the first constraint. In particular $I \backslash\{3\} \cup F \in X_{1}$. Finally, we also remark that $I \cup\{5\} \in X_{1}$ and $I \backslash\{3\} \cup\{5\} \in X_{2}$. Together this means that

$$
I \cup\{5\} \in X_{1} \quad \text { and } I \backslash\{3\} \cup\{5,6,8\} \in X_{1} .
$$

By taking half of the sum of the incidence vectors of these two sets, this shows that the point $x$ with coordinates

$$
x_{1}=1, x_{3}=\frac{1}{2}, x_{4}=1, x_{5}=1, x_{6}=\frac{1}{2}, x_{7}=1, x_{8}=\frac{1}{2}
$$

belongs to $\operatorname{conv}\left(X_{1}\right)$. We also have that

$$
I \cup\{5,6,8\} \in X_{2} \quad \text { and } I \backslash\{3\} \cup\{5\} \in X_{2} .
$$

By taking again half of the sum of the incidence vectors of these two sets, we obtain the same point $x$ defined by (33). This shows that $x \in \operatorname{conv}\left(X_{2}\right)$. Therefore $x \in \operatorname{conv}\left(X_{1}\right) \cap \operatorname{conv}\left(X_{2}\right)$ and $s(I) \geq \frac{1}{2}$.

The example illustrates a constructive way to compute a bound on the strength of a given inequality (28). In the remainder of this section we formalize this construction.

We consider an inequality (28) that is valid for $X_{1} \cap X_{2}$. In order to state the main result about its strength, the following assumption is made.

Assumption 1 There exist $F \subset N_{-} \backslash\left(I \cup I^{C}\right)$ and $i_{0}$ such that $I \cup F \in X_{2}$ and $I \backslash\left\{i_{0}\right\} \cup F \in X_{1} \cap X_{2}$. 
If the first part of Assumption 1 is not satisfied, it simply means that the inequality (28) is also valid for $X_{2}$. In this case, the strength would be trivially 0 . The second part of Assumption 1 requires that the inequality is sufficiently tight. This condition is however not always satisfied. The following definition is related to the choices of $F$ and $i_{0}$ refered to Assumption 1.

Definition 3 We define $H \subset F, F \neq H$ such that $I \cup H \in X_{1}$, with $H$ being possibly empty.

Let $p$ denote the cardinality of the largest partition of $F \backslash H$, say $\left(\bar{G}_{1}^{1}, \ldots, \bar{G}_{p}^{1}\right)$ such that $I \cup H \cup G_{i}^{1} \in X_{1}$ for all $i$ with $G_{i}^{1}=(F \backslash H) \backslash \bar{G}_{i}^{1}$.

Let $q$ denote the cardinality of the smallest partition of $F \backslash H$, say $\left(\bar{G}_{1}^{2}, \ldots, \bar{G}_{q}^{2}\right)$ such that $I \backslash\left\{i_{0}\right\} \cup H \cup G_{i}^{2} \in X_{2}$ for all $i$ with $G_{i}^{2}=(F \backslash H) \backslash \bar{G}_{i}^{2}$.

Let $T$ be a bipartite graph, $T=\left(\left(V_{1}, V_{2}\right), E\right)$, with $p$ nodes in $V_{1}$ and $q$ nodes in $V_{2}$. There is an edge between $i \in V_{1}$ and $j \in V_{2}$ whenever $\bar{G}_{i}^{1} \cap \bar{G}_{j}^{2} \neq \emptyset$.

Theorem 3 Let (28) be a valid inequality for the intersection of two knapsacks. We have, under Assumption 1, that

$$
s\left(I, I^{C}\right) \geq \kappa,
$$

with $\kappa=\max _{l} \frac{\left|V_{1}^{l}\right|}{\left|V_{1}^{l}\right|+\left|V_{2}^{l}\right|}$, where $T^{l}$ is a connected component of the bipartite graph $T$ of Definition 3.

Proof: Let $T^{d}=\left(\left(V_{1}^{d}, V_{2}^{d}\right), E^{d}\right)$ be a connected component of $T$ for which $\kappa=\frac{\left|V_{1}^{d}\right|}{\left|V_{1}^{d}\right|+\left|V_{2}^{d}\right|}$. Let $V_{1}^{d}=\left\{v_{1}, \ldots, v_{s}\right\}$ and $V_{2}^{d}=\left\{w_{1}, \ldots, w_{t}\right\}$. Each element of $V_{1}^{d}$ is in correspondence with one set $\bar{G}_{i}^{1}$ of the partition introduced in Definition 3 . We consider the union of these sets taken over the vertices from $V_{1}^{d}$,

$$
E=\bigcup_{i \in V_{d}^{1}} \bar{G}_{i}^{1}
$$

Next we show that the point $x$ with coordinates

$$
\begin{array}{ll}
x_{i}=1 & \text { for all } i \in I \backslash\left\{i_{0}\right\} \\
x_{i_{0}}=\frac{\left|V_{1}^{d}\right|}{\left|V_{1}^{d}\right|+\left|V_{2}^{d}\right|} & \text { for all } i \in H \\
x_{i}=1 & \text { for all } i \in E \\
x_{i}=\frac{1}{\left|V_{1}^{d}\right|+\left|V_{2}^{d}\right|} &
\end{array}
$$

belongs to $\operatorname{conv}\left(X_{1}\right) \cap \operatorname{conv}\left(X_{2}\right)$.

We first show that $x \in \operatorname{conv}\left(X_{1}\right)$. It is indeed a convex combination of the incidence vectors of the sets

$$
I \backslash\left\{i_{0}\right\} \cup F, I \cup H \cup G_{v_{1}}^{1}, \ldots, I \cup H \cup G_{v_{s}}^{1},
$$

with coefficients $\frac{\left|V_{2}^{d}\right|}{\left|V_{1}^{d}\right|+\left|V_{2}^{d}\right|}, \frac{1}{\left|V_{1}^{d}\right|+\left|V_{2}^{d}\right|}, \ldots, \frac{1}{\left|V_{1}^{d}\right|+\left|V_{2}^{d}\right|}$, respectively. The sets listed in (35) all belong to $X_{1}$ by Assumption 1 and Definition 3. 
We now show that $x \in \operatorname{conv}\left(X_{2}\right)$. To do this, we first notice that $E^{\prime}$ defined as

$$
E^{\prime}=\bigcup_{i \in V_{d}^{2}} \bar{G}_{i}^{2}
$$

is exactly equal to $E$ defined in (34). Indeed suppose that $E \neq E^{\prime}$. Then there exists $j \in F \backslash H$ such that $j \in \bar{G}_{i}^{1}$ for some $i \in V_{1}^{d}$ and

$$
j \notin \bar{G}_{k}^{2} \quad \text { for all } k \in T_{2}^{d} .
$$

On the other hand since $\left(\bar{G}_{1}^{2}, \ldots, \bar{G}_{q}^{2}\right)$ is a partition of $F \backslash H$, there exists a set $\bar{G}_{k}^{2}$ that contains $j$. Therefore the vertex corresponding to $G_{k}^{2}$ should be connected to that corresponding to $\bar{G}_{i}^{1}$, and hence to $V_{1}^{d}$. This contradicts (36). It follows that $x$ is a convex combination of the incidence vectors of the sets

$$
I \cup F, I \backslash\left\{i_{0}\right\} \cup H \cup G_{w_{1}}^{2}, \ldots I \backslash\left\{i_{0}\right\} \cup H_{\cup} G_{w_{t}}^{2},
$$

with coefficients $\frac{\left|V_{1}^{d}\right|}{\left|V_{1}^{d}\right|+\left|V_{2}^{d}\right|}, \frac{1}{\left|V_{1}^{d}\right|+\left|V_{2}^{d}\right|}, \ldots, \frac{1}{\left|V_{1}^{d}\right|+\left|V_{2}^{d}\right|}$ respectively. The sets listed in (37) all belong to $X_{2}$ by Assumption 1 and Definition 3.

Theorem 3 relates the strength of an incomplete set inequality with the size of partitions in a certain graph. The construction itself is very general and only depends on the instance. This is why the result can be applied whenenver Assumption 1 holds. It is easy to modify the construction so as to apply to general inequalities for the intersection of two convex hulls. Returning to the incomplete set inequalities, from the initial example one might get the impression that the strength of this family of inequalities is always at least 0.5 . This, however is not true as the next example shows. This example also illustrates the construction elucidated in the proof of Theorem 3.

Example Let us consider the set

$$
\begin{array}{r}
X=\left\{x \in\{0,1\}^{7}: 7 x_{1}+7 x_{2}+2 x_{3}+2 x_{4}+2 x_{5}+2 x_{6}+2 x_{7} \leq 17\right. \\
\left.7 x_{1}+7 x_{2}-3 x_{3}-3 x_{4}-3 x_{5}-3 x_{6}-3 x_{7} \leq 0\right\},
\end{array}
$$

and the valid inequality $x_{1}+x_{2} \leq 1$. Defining $F=\{3,4,5,6,7\}$ and $i_{0}=\{1\}$ shows that Assumption 1 holds. For Definition 3, we set $H=\{3\}$. The first partition is obtained by one set $\bar{G}_{1}^{1}=F \backslash H$, with $I \cup H \cup \emptyset \in X_{1}$. The second partition consists of two sets $\bar{G}_{1}^{2}=\{4,5\}$ and $\bar{G}_{2}^{2}=\{6,7\}$, with $I \backslash\left\{i_{0}\right\} \cup H \cup G_{i}^{2} \in$ $X_{2}, i=1,2$. The bipartite graph has one node in $V_{1}$ and two nodes in $V_{2}$, which are both connected to the node in $V_{1}$. We have one connected component with $\left|V_{1}\right| /\left(\left|V^{1}\right|+\left|V^{2}\right|\right)=1 / 3$. By Theorem 3, this shows that $x_{1}+x_{2} \leq 1$ has a strength of at least $1 / 3$. It can be checked that it is also the exact strength of the inequality.

The value of Theorem 3 is that it allows us to compute a bound for the strength of an incomplete set inequality for every instance. Note that this is nontrivial as the strength is defined as the value of an optimization problem that 
requires the enumeration of all the facets of the two knapsack polyhedra. This is impossible to do for more than $15-20$ variables. Next we want to verify whether Theorem 3 is applicable in many cases and then use it to compute bounds on the strength. Note that in order to apply Theorem 3, it is necessary that Assumption 1 holds! For our tests we have generated randomly two instances with 40 and 50 variables respectively. Since it is too expensive to enumerate all possible incomplete sets, we decided to generate randomly one million incomplete sets for each instance. For each generated set, we first check whether its residue and its excess are in a reasonable interval (to avoid computing irrelevant sets). We then compute the corresponding incomplete set inequality and try to apply Theorem 3 in order to obtain a bound on the strength. Among the obtained inequalities, some are valid for the knapsack $X_{2}$ only. We also get rid of them. The results for the remaining inequalities are reported in Table 3. For each

\begin{tabular}{lcccccc} 
strength & $?$ & 0.5 & 0.6 & 0.667 & 0.75 & 0.8 or more \\
\hline $\begin{array}{l}\text { Instance 1 (40 var) } \\
13 \%\end{array}$ & $36 \%$ & $25 \%$ & $16 \%$ & $8 \%$ & $2 \%$ \\
11719 inequalities tested & & & & & \\
\hline $\begin{array}{l}\text { Instance 2 (50 var) } 3 \% \\
18128 \text { inequalities tested }\end{array}$ & $44 \%$ & $9 \%$ & $32 \%$ & $12 \%$ & $0 \%$ \\
\hline
\end{tabular}

Table 3: Computing of a bound on the strength using Theorem 3

instance, we report on the proportion of inequalities reaching a given computed bound. The column "?" indicates the proportion of inequalities for which Theorem 3 cannot be applied and a refined version of the theorem would be needed. We conclude that in the vast majority of cases Assumption 1 holds. There are only approximately $10 \%$ of the inequalities that we detected to which Theorem 3 did not apply. Furthermore we also see that the bound computed by Theorem 3 is in all the cases at least equal to 0.5 .

\section{Extension to the mixed case}

In [6], the authors show that valid inequalities for the binary knapsack can be extended to the case where one continuous variable appears. In this section, we show that the incomplete set inequalities can also be extended to the mixed case by lifting the continuous variables. We consider the models

$$
\begin{aligned}
& X_{1}=\left\{(x, s, t) \in\{0,1\}^{n} \times \mathbb{R}_{+}^{2}: \sum_{i=1}^{n} a_{i} x_{i} \leq a_{0}+s\right\}, \\
& X_{2}=\left\{(x, s, t) \in\{0,1\}^{n} \times \mathbb{R}_{+}^{2}: \sum_{i=1}^{n} b_{i} x_{i} \leq b_{0}+t\right\}, \\
& X=\left\{(x, s, t) \in\{0,1\}^{n} \times \mathbb{R}_{+}^{2}:(x, s, t) \in X_{1} \cap X_{2}\right\} .
\end{aligned}
$$


We also define the restricted sets $X_{i}^{r}=\left\{x \in\{0,1\}^{n}:(x, 0,0) \in X_{i}\right\}$ for $i=1,2$ and similarly for $X$. We first fix $s=t=0$ and find a valid incomplete set inequality

$$
\sum_{i \in I} x_{i}-\sum_{i \in I^{C}} x_{i} \leq|I|-1
$$

for the restricted set $X^{r}$. We also consider its associated residue $r(I)$ and excess $e(I)$ as defined in Section 3. In the following we consider that (38) is not valid for the single knapsacks $X_{1}^{r}$ and $X_{2}^{r}$ taken separately. We now show how to lift the variables $s$ and $t$ in (38) and obtain the valid inequality

$$
\sum_{i \in I} x_{i}-\sum_{i \in I^{C}} x_{i}+\alpha s+\beta t \leq|I|-1
$$

for the set $X$. To do this we define a lifting function as proposed in [5],

$$
\begin{aligned}
\phi(u, v)=\min & |I|-1-\sum_{i \in I} x_{i}+\sum_{i \in I^{C}} x_{i} \\
\text { s.t. } & \sum_{i \in N} a_{i} x_{i} \leq a_{0}+u \\
& \sum_{i \in N_{+}} b_{i} x_{i}-\sum_{i \in N_{-}} b_{i} x_{i} \leq b_{0}+v \\
& x_{i} \in\{0,1\}^{n} .
\end{aligned}
$$

A pair $(\alpha, \beta)$ is a valid pair of lifting coefficients if and only if

$$
\alpha u+\beta v \leq \phi(u, v)
$$

for all $(u, v) \in \mathbb{R}_{+}^{2}$.

Lemma 1 If there exists at least one solution $x \in X^{r}$ such that $\sum_{i \in I} x_{i}-$ $\sum_{i \in I^{C}} x_{i}=|I|-1$, then

$$
\phi(u, v) \in\{0,-1\}
$$

for all $(u, v) \in \mathbb{R}_{+}^{2}$.

Proof: Let us fix $u, v \geq 0$. Two cases occur.

1. $\sum_{i \in I} a_{i}>a_{0}+u$. In this case, the solution provided by the hypothesis gives a value of 0 , while it is clearly impossible to achieve less than that.

2. $\sum_{i \in I} a_{i} \leq a_{0}+u$. In this case, it may be possible to have a value of $\phi$ lower than 0 . It can only be -1 by setting $x_{i}=1$ for all $i \in I$.

The question is now to find the points $(u, v)$ for which the value of $\phi$ goes from 0 to -1 . These points determine a finite set of linear inequalities which define the valid lifting coefficients. We next reintroduce two notations used earlier to compute the strength of an inequality. 
Definition 4 We define the sets $F$ and $H$ as

$$
\begin{gathered}
F=\arg \min \left\{a(S):|b(S)| \geq e(I), S \subseteq N_{-} \backslash\left(I \cup I^{C}\right)\right\}, \\
H=\arg \min \left\{b(S): a(S) \leq r(I), S \subseteq N_{-} \backslash\left(I \cup I^{C}\right)\right\} .
\end{gathered}
$$

The set $F$ and $H$ determine thresholds from which the function $\phi$ takes the value -1 .

Observation 2 (i) For all $u \geq a(F)-r(I)$ and $v \geq 0$,

$$
\phi(u, v)=-1 .
$$

(ii) For $u \geq 0, v \geq e(I)-|b(H)|$,

$$
\phi(u, v)=-1 .
$$

Proof: For $u \geq a(F)-r(I), v \geq 0$, it turns out that $I \cup F$ is an optimal solution of (40). This proves part (i).

Similarly for $u \geq 0, v \geq e(I)-|b(H)|, I \cup H$ is an optimal solution of (40).

Observation 2 provides bounds for the lifting coefficients $\alpha$ and $\beta$.

Lemma 2 Necessary conditions for lifting coefficients $\alpha$ and $\beta$ of (39) are

$$
\begin{aligned}
& \alpha \leq \frac{-1}{a(F)-r(I)} \\
& \beta \leq \frac{-1}{e(I)-|b(H)|} .
\end{aligned}
$$

Proof: First remark that, by definition of $F$, we always have $a(F)>r(I)$ since (38) is valid for $X^{r}$. Similarly we also have $|b(H)|<e(I)$. Therefore the inequalities given in the lemma are always well defined. To find them, we express conditions (41) for $(u, v)=(a(F)-r(I), 0)$ and $(u, v)=(0, e(I)-|b(H)|)$.

As one might expect, the condition on $\alpha, \beta$ given in Lemma 2 are in general not sufficient to describe a valid lifting pair.

Theorem 4 For each set $G \subseteq N_{-} \backslash\left(I \cup I^{C}\right)$, we define an inequality $\mathcal{I}_{G}$

$$
(a(G)-r(I))^{+} \alpha+(e(I)-b(G))^{+} \beta \leq-1,
$$

where $a^{+}=\max \{a, 0\}$. The set $\Pi$ of valid lifting coefficients is a polyhedron defined as

$$
\begin{gathered}
\Pi=\left\{(\alpha, \beta):(\alpha, \beta) \text { satisfy } \mathcal{I}_{G} \text { for all } G \text { with } a(G)>r(I) \text { and } b(G)<e(I)\right. \\
\qquad \leq \frac{-1}{a(F)-r(I)} \\
\left.\quad \beta \leq \frac{-1}{e(I)-|b(H)|} \quad\right\} .
\end{gathered}
$$


Proof: We need to show that for all $(\alpha, \beta) \in \Pi$ and all $(u, v) \in \mathbb{R}_{+}^{2}$, we have

$$
\alpha u+\beta v \leq \phi(u, v) .
$$

Let us fix $(\alpha, \beta) \in \Pi$ and $(u, v) \in \mathbb{R}_{+}^{2}$. If $\phi(u, v)=0$, we clearly have the condition since $\alpha, \beta<0$. Let us now suppose that $\phi(u, v)=-1$. By definition of $\phi$, there exists a set $K \subseteq N_{-} \backslash\left(I \cup I^{C}\right)$, optimal for (40) such that $a(I \cup$ $K) \leq a_{0}+u$ and $b(I \cup K) \leq b_{0}+v$. We just need to prove inequality (42) for $\bar{u}=\left(a(I \cup K)-a_{0}\right)^{+} \leq u$ and $\bar{v}=\left(b(I \cup K)-b_{0}\right)^{+} \leq v$. Notice that $(\bar{u}, \bar{v})=\left((a(K)-r(I))^{+},(e(I)-|b(K)|)^{+}\right)$. Three cases occur. If $\bar{u}, \bar{v}>0$, then the inequality $\alpha u+\beta v \leq \phi(u, v)$ appears in the representation of the polyhedron. If $\bar{u}=0$, then $\bar{v}>0$ since the inequality to be lifted is valid for $X^{r}$, and $\bar{v} \geq e(I)-|b(H)|$ by definition of $H$. Therefore (42) is satisfied by the condition on $\beta$. Similarly if $\bar{v}=0$, the inequality is satisfied by the condition on $\alpha$, by definition of $F$.

Example 5 Consider the set

$$
\begin{aligned}
& X=\left\{(x, s, t) \in\{0,1\}^{5} \times \mathbb{R}_{+}^{2}: 7 x_{1}+8 x_{2}+6 x_{3}+9 x_{4}+10 x_{5} \leq 22+s\right. \\
& \left.4 x_{1}+3 x_{2}-x_{3}-3 x_{4}-5 x_{5} \leq t \quad\right\} .
\end{aligned}
$$

If we fix $s=t=0$, we find that $x_{1}+x_{2} \leq 1$ is a valid inequality for the restricted set. To lift $s$ and $t$, we consider the lifting function

$$
\begin{aligned}
\phi(u, v)=\min & 1-x_{1}-x_{2} \\
\text { s.t. } & 7 x_{1}+8 x_{2}+6 x_{3}+9 x_{4}+10 x_{5} \leq 22+u \\
& 4 x_{1}+3 x_{2}-x_{3}-3 x_{4}-5 x_{5} \leq v \\
& x_{i} \in\{0,1\} .
\end{aligned}
$$

The lifting function is depicted in Figure 3. The function takes the value 0 in the white region and the value -1 in the gray region. The points marked with a circle determine linear inequalities that define the polyhedron of valid lifting coefficients. These five inequalities are

$$
\begin{aligned}
& 6 \beta \leq-1 \\
& 2 \alpha+4 \beta \leq-1 \\
& 3 \alpha+2 \beta \leq-1 \\
& 9 \alpha+\beta \leq-1 \\
& 12 \alpha \quad \leq-1 .
\end{aligned}
$$

The polyhedron they describe is shown in Figure 4 . It has two vertices $(-2 / 9,-1 / 6)$ and $(-1 / 12,-3 / 8)$ which are therefore the two pairs of maximal lifting coefficients. They lead to the valid inequalities

$$
\begin{gathered}
x_{1}+x_{2}-\frac{2}{9} s-\frac{1}{6} t \leq 1 \\
x_{1}+x_{2}-\frac{1}{12} s-\frac{3}{8} t \leq 1
\end{gathered}
$$




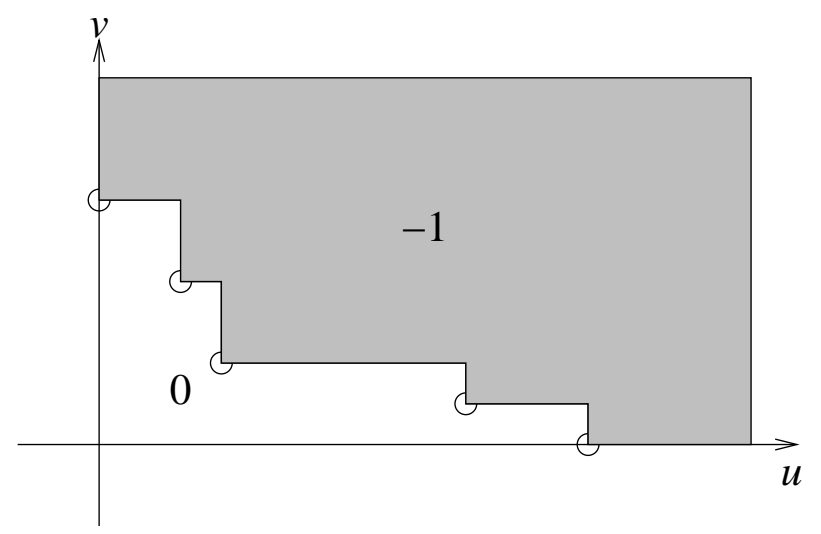

Figure 3: The value of the lifting function $\phi(u, v)$

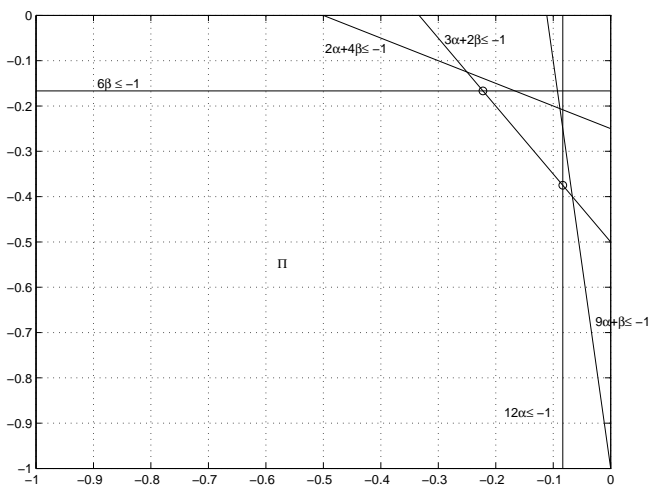

Figure 4: The polyhedron $\Pi$ of valid lifting coefficients determined by the linear inequalities obtained at the circled points of Figure 3 
In the following example we indicate how lifted incomplete set inequalities can be generated from a simplex tableau.

Example 6 We consider the problem

$$
\begin{aligned}
& \min \quad x_{1}+2 x_{2}+3 x_{3}+4 x_{4}+5 x_{5}+6 x_{6} \\
& \text { s.t. } 17 x_{1}+17 x_{2}+24 x_{3}+13 x_{4}+21 x_{5}+15 x_{6}+s_{1} \leq 54 \\
& -14 x_{1}-19 x_{2}-13 x_{3}-18 x_{4}-13 x_{5}-29 x_{6} \quad+s_{2} \quad \leq-53 \\
& +7 x_{1}+20 x_{2}+18 x_{3}+19 x_{4}+8 x_{5}+16 x_{6} \quad+s_{3} \quad \leq 44 \\
& -12 x_{1}-7 x_{2}-11 x_{3}-6 x_{4}-10 x_{5}-14 x_{6} \quad+s_{4} \leq-30 \\
& x_{1}, \quad x_{2}, \quad x_{3}, \quad x_{4}, \quad x_{5}, \quad x_{6} \quad \in\{0,1\}
\end{aligned}
$$

An optimal LP solution $x^{*}$ for (43) is

$$
x_{1}^{*}=x_{2}^{*}=1, x_{3}^{*}=0.28, x_{6}^{*}=0.56, s_{1}^{*}=4.74, s_{3}^{*}=2.88 .
$$

We consider the optimal LP tableau. In particular, the first two rows of the optimal LP tableau can be written in integer form as

$$
\begin{aligned}
152 x_{1}-63 x_{2}+137 x_{3}-78 x_{4}+108 x_{5}+14 s_{2}-29 s_{4} & =128 \\
-2 x_{1}+118 x_{2}+120 x_{4}+13 x_{5}+137 x_{6}-11 s_{2}+13 s_{4} & =193
\end{aligned}
$$

We relax $14 s_{2}$ in (44) and aggregate $29 s_{4}$ into one continuous variable $t_{1}$. Similarly we relax $13 s_{4}$ in (45) and aggregate $11 s_{2}$ into one continuous variable $t_{2}$. This yields the relaxed problem

$$
\begin{aligned}
152 x_{1}-63 x_{2}+137 x_{3}-78 x_{4}+108 x_{5} & \leq 128+t_{1} \\
-2 x_{1}+118 x_{2}+120 x_{4}+13 x_{5}+137 x_{6} & \leq 193+t_{2} .
\end{aligned}
$$

In order to obtain a canonical form, i.e. all coefficients of (46) are nonnegative, we introduce the complemented variables $\bar{x}_{2}=1-x_{2}$ and $\bar{x}_{4}=1-x_{4}$. We finally consider the modified relaxed problem

$$
\begin{array}{rlr}
152 x_{1}+63 \bar{x}_{2}+137 x_{3}+78 \bar{x}_{4}+108 x_{5} & \leq 128+t_{1} \\
-2 x_{1}-118 \bar{x}_{2}-120 \bar{x}_{4}+13 x_{5}+137 x_{6} & \leq-45+t_{2} .
\end{array}
$$

We first fix $t_{1}=t_{2}=0$ and consider the restricted set. Our incomplete set is $I=\{6\}$ with $r(I)=128$ and $e(I)=182$. We see that in order to compensate for such an excess, we need to set $\bar{x}_{2}=\bar{x}_{4}=1$ but this exceeds the residue in (47). Hence $x_{6} \leq 0$ is a valid inequality for the restricted set. We now need to lift the inequality. Therefore we consider the function $\phi\left(t_{1}, t_{2}\right)=\min \left\{-x_{6} \mid(47),(48), x_{i} \in\{0,1\}\right\}$. We obtain

$$
\phi\left(t_{1}, t_{2}\right)= \begin{cases}-1 & \text { if } t_{1} \geq 0, t_{2} \geq 62 \\ -1 & \text { if } t_{1} \geq 13, t_{2} \geq 0 \\ 0 & \text { otherwise }\end{cases}
$$


This implies that a pair of valid maximal lifting coefficients is $\left(\frac{-1}{13}, \frac{-1}{62}\right)$ for $t_{1}$ and $t_{2}$ respectively. If we substitute back $t_{1}=29 s_{4}$ and $t_{2}=11 s_{2}$, we obtain the valid inequality

$$
x_{6} \leq \frac{29}{13} s_{4}+\frac{11}{62} s_{2}
$$

which cuts off $x^{*}$.

\section{Acknowledgements}

We would like to thank Laurence Wolsey for his comments on this manuscript. We also would like to thank one anonymous referee for his helpful remarks and for giving the idea of an improved version of the proof of Theorem 1. This work was supported by the European ADONET Program 504438.

\section{References}

[1] E. Balas. Facets of the knapsack polytope. Mathematical Programming, 8:146-164, 1975.

[2] Elena Fernández and Kurt Jørnsten. Partial cover and complete cover inequalities. Operations Research Letters, 15:19-33, 1994.

[3] O. Günlük and Y. Pochet. Mixing mixed-integer inequalities. Mathematical Programming, 90:429-457, 2001.

[4] P. L. Hammer, E. L. Johnson, and U. N. Peled. Facets of regular 0-1 polytopes. Mathematical Programming, 8:179-206, 1975.

[5] Q. Louveaux and L. A. Wolsey. Lifting, superadditivity, mixed integer rounding and single node flow sets revisited. Quarterly Journal of the Belgian, French and Italian Operations Research Societies, 1:173-207, 2003.

[6] H. Marchand and L. A. Wolsey. The 0-1 knapsack problem with a single continuous variable. Mathematical Programming, 85:15-33, 1999.

[7] A. Martin and R. Weismantel. The intersection of knapsack polyhedra and extensions. In R.E. Bixby, E.A. Boyd, and R.Z. Rios-Mercado, editors, Proc. IPCO 98, pages 243-256. Lecture Notes in Computer Science 1412, Springer, Berlin, 1998.

[8] Robert Weismantel. On the 0/1 knapsack polytope. Mathematical Programming, 77:49-68, 1997.

[9] L. A. Wolsey. Faces of linear inequalities in 0-1 variables. Mathematical Programming, 8:165-178, 1975. 